\title{
Serologic features of cohorts with variable genetic risk for systemic lupus erythematosus
}

\author{
Jyotsna Bhattacharya ${ }^{1}$, Karalyn Pappas², Bahtiyar Toz ${ }^{3}$, Cynthia Aranow¹, Meggan Mackay ${ }^{1}$, Peter K. Gregersen ${ }^{4}$, \\ Ogobara Doumbo ${ }^{5}$, Abdel Kader Traore ${ }^{6}$, Martin L. Lesser ${ }^{7}$, Maureen McMahon ${ }^{8}$, Tammy Utset ${ }^{9}$, Earl Silverman ${ }^{10}$, \\ Deborah Levy ${ }^{10}$, William J. McCune ${ }^{11}$, Meenakshi Jolly ${ }^{12}$, Daniel Wallace ${ }^{13}$, Michael Weisman ${ }^{13}$, Juanita Romero-Diaz ${ }^{14}$ \\ and Betty Diamond ${ }^{1 *}$
}

\begin{abstract}
Background: Systemic lupus erythematosus (SLE) is an autoimmune disease with genetic, hormonal, and environmental influences. In Western Europe and North America, individuals of West African descent have a 3-4 fold greater incidence of SLE than Caucasians. Paradoxically, West Africans in sub-Saharan Africa appear to have a low incidence of SLE, and some studies suggest a milder disease with less nephritis. In this study, we analyzed sera from African American female SLE patients and four other cohorts, one with SLE and others with varying degrees of risk for SLE in order to identify serologic factors that might correlate with risk of or protection against SLE.

Methods: Our cohorts included West African women with previous malaria infection assumed to be protected from development of SLE, clinically unaffected sisters of SLE patients with high risk of developing SLE, healthy African American women with intermediate risk, healthy Caucasian women with low risk of developing SLE, and women with a diagnosis of SLE. We developed a lupus risk index (LRI) based on titers of IgM and IgG anti-double stranded DNA antibodies and levels of C1q.
\end{abstract}

Results: The risk index was highest in SLE patients; second highest in unaffected sisters of SLE patients; third highest in healthy African-American women and lowest in healthy Caucasian women and malaria-exposed West African women.

Conclusion: This risk index may be useful in early interventions to prevent SLE. In addition, it suggests new therapeutic approaches for the treatment of SLE.

\section{Background}

Systemic lupus erythematosus (SLE) is a chronic systemic autoimmune disease characterized by defects in B cell tolerance leading to the production of multiple autoantibodies. In particular, SLE is characterized by high affinity IgG anti-nuclear autoantibodies including antidouble stranded (ds) DNA antibodies.

Anti-dsDNA antibodies are found in $70 \%$ of patients, are pathogenic and are frequently used to monitor disease activity (Pavlovic et al. 2010; Linnik et al. 2005). Published data demonstrate a 'preclinical' period of disease characterized by

\footnotetext{
* Correspondence: bdiamond@northwell.edu

${ }^{1}$ The Feinstein Institute for Medical Research, Center for Autoimmune, Musculoskeletal and Hematopoietic Diseases, 350 Community Dr, Manhasset, NY 11030, USA

Full list of author information is available at the end of the article
}

the presence of IgG autoantibodies with increasing titers and number of auto- specificities heralding the onset of clinical SLE (Deane and El-Gabalawy 2014; Arbuckle et al. 2003). However, reports of elevated autoantibody titers in first degree relatives suggest that the presence of autoantibodies alone does not confer disease (Ramos et al. 2010).

While the etiology SLE is not known, data suggest that susceptibility requires both a genetic predisposition and environmental triggers. The genetic predisposition is highlighted by the observed familial clustering of SLE and a concordance rate of approximately $30 \%$ in identical twins. Over 50 risk alleles for SLE have been identified and disease severity and age of onset relates, in part, to the number of risk alleles present in an individual (Teruel and Alarcon-Riquelme 2016). Disease is $8-10$

(C) The Author(s). 2018 Open Access This article is distributed under the terms of the Creative Commons Attribution 4.0 International License (http://creativecommons.org/licenses/by/4.0/), which permits unrestricted use, distribution, and 
times more prevalent in women than men and 3-4 times more prevalent in women of African descent in Europe or North America than in Caucasian women (Gilkeson et al. 2011). In Caribbean populations, an increasing number of African genes rather than genetic admixture is a risk factor for disease (Molokhia et al. 2003; Molokhia and McKeigue 2000). The prevalence of SLE in West African women is not fully established, but several studies have suggested a lower prevalence in African countries (George and Ogunbiyi 2005; McGill and Oyoo 2002; Molokhia et al. 2001). Moreover, disease manifestations appear to be less severe in West African patients, with a lower incidence of renal disease (Zomalheto et al. 2014). It is reasonable to assume that the genetic predisposition to SLE is at least as high in West Africans as in African-Americans and Afro-Caribbeans and the discrepancy in disease prevalence reflects the impact of environmental factors (Molokhia et al. 2001).

Malaria, an endemic infection in sub-Saharan Africa, has long been suggested to mitigate the impact of SLE (Greenwood 1968). That malaria protects against development of SLE has been clearly demonstrated in spontaneously lupus-prone mice (Greenwood et al. 1970). Because it is frequently fatal, it likely has exerted significant pressure on the genome, resulting in the retention of alleles that diminish the severity of infection. Several risk alleles for SLE protect against severe malaria infection. The FcRllb risk allele for SLE (T232) leads to a non-functional molecule which cannot move through the plasma membrane to associate with the $B$ cell receptor (Floto et al. 2005). Decreased inhibitory function associated with this risk allele results in increased B cell and myeloid cell activation. While this may increase risk for SLE, it can be beneficial for a response to infection. In humans, FcRllb T232 increases phagocytosis of P. falciparum by monocyte-derived macrophages in vitro (Clatworthy et al. 2007). Moreover, FcRllb-deficient mice are resistant to severe disease following infection with Plasmodium Chabaudi (Clatworthy et al. 2007). Notably, polymorphisms predisposing to low TNF levels protect against cerebral malaria. Several lupus-prone strains show reduced levels attributable to a promoter region polymorphism in the $N Z B, B X S B$ and $M R L$ strains. (Jiang et al. 1999; Pritchard et al. 2000) and administering TNF to these mice can prevent the onset of SLE.

The repertoire of immunocompetent B cells develops as a consequence of tolerance mechanisms that censor a majority of autoreactive $\mathrm{B}$ cells during their maturation process. Approximately $75 \%$ of immature B cells have an autoreactive BCR compared to $20 \%$ of naïve immunocompetent B cells (Hoffman et al. 2016). These B cells are critical for immune homeostasis as they produce IgM antibodies capable of binding to and removing apoptotic debris in a non-immunogenic fashion (Gronwall et al.
2012). Lack of these autoreactive IgM antibodies results in uptake of apoptotic material in dendritic cells (DCs) and DC activation (Ehrenstein et al. 2000). In NZB/W lupusprone mice, production of pathogenic IgG anti-dsDNA autoantibodies coincides with diminished production of IgM autoantibodies, and administration of IgM antidsDNA autoantibodies prevents development of renal disease in mice (Werwitzke et al. 2005).

Although malaria infection may protect against the development of SLE in spontaneous murine models of SLE, an association between malarial infection and autoantibodies is well recognized (Daniel-Ribeiro and Zanini 2000). Many of the autoantibodies present in malaria patients are IgM and are not known to be pathogenic (Wozencraft et al. 1990). The ability of IgM autoantibodies to maintain immune quiescence occurs through a C1q dependent mechanism (Gronwall and Silverman 2014).

$\mathrm{C} 1 \mathrm{q}$ is a complement component that is important in clearance of apoptotic debris and promotes immune tolerance through regulation of immune cell differentiation and cytokine release (Son et al. 2015). Ninety percent of individuals with severe hereditary $\mathrm{C} 1 \mathrm{q}$ deficiency have SLE (Manderson et al. 2004).

We hypothesized that an enhanced ratio of IgG:IgM anti-DNA antibodies and a diminished level of C1q would predispose to SLE. We further hypothesized that exposure to malaria results in increased titers of protective IgM autoantibodies and increases in $\mathrm{Clq}$ that retard or prevent onset of SLE in genetically predisposed individuals.

We, therefore, evaluated IgM and IgG anti-dsDNA antibody titers and assessed C1q levels in women with varying risk for SLE based on genetic risk and malaria exposure: African-American SLE patients (SLE); healthy Caucasian women (CHC); healthy African-American women (AAHC); unaffected sisters of SLE patients (SIS); and women from Mali with a history of malaria infection (MAL). We generated a lupus risk index (LRI) based on serum IgG:IgM antiDNA antibody ratio and $\mathrm{Clq}$ level. The a priori hypothesis was that the LRI would be lowest in $\mathrm{CHC}$, then increase through groups MAL, AAHC, SIS, and SLE, in that order. The development of an LRI may prove useful in following at risk individuals over time to identify those that may profit from early intervention and diagnosed SLE patients who might be at risk for an impending flare.

\section{Methods \\ Samples}

Serum samples were obtained from 40 Malian women, (MAL) aged 18 to 65. Inclusion criteria included a known history of malaria infection, no history of autoimmune disease or first degree relative with autoimmune disease and no known infection with HIV. Additional serum samples were obtained from 51 SLE patients of African American descent (SLE). All SLE subjects met 
1997 ACR revised criteria and were enrolled in the prospective SLE cohort at the Feinstein Institute. Serum samples from 80 healthy African American women (AAHC), age 20 to 68, with no use of immunosuppressive agents in the year prior, and 16 Caucasian healthy controls $(\mathrm{CHC})$, age 28 to 50, were purchased from BioreclamationIVT. Serum from 98 unaffected sisters of SLE patients (SIS), age 14-46, was obtained from the Feinstein Institute SisSLE cohort. The SIS cohort included 67 Caucasian, 11 Hispanics, 7 African-Americans and 12 Asians, (one unknown). The study was approved by the Institutional Review Board at the Northwell Health, Manhasset, NY and the Comité d'Ethique de la FMPOS, Bamako, Mali.

\section{dsDNA ELISA}

To detect IgM and IgG anti-dsDNA antibodies, 96-well plates (Costar, 3690, Corning, Kennenbunk, ME) were coated with calf thymus DNA that had been filtered through a 0.45 um cellulose filter (Millipore, Darmstadt, Germany) to remove ssDNA (\#2618, Calbiochem, San Diego, CA) at $2 \mu \mathrm{g} / \mathrm{ml}$ in PBS. Plates were dry-coated overnight at $370 \mathrm{C}$ and blocked in 3\% FBS/PBS for $1 \mathrm{~h}$ at room temperature (RT). Plates were washed 3 times and then incubated with serum samples diluted 1:100 in $0.3 \% \mathrm{FBS} / \mathrm{PBS}$ and assayed in triplicate. Plates were washed 5 times in PBS 0.05\% Tween, and then incubated with secondary anti-IgM or IgG alkaline phosphatase conjugated antibodies (SouthernBiotech, Birmingham, $\mathrm{AL}$ ) diluted 1:000 in $0.3 \% \mathrm{FBS} / \mathrm{PBS}$ for $1 \mathrm{~h}$ at $370 \mathrm{C}$, washed 3 times, and developed with alkaline phosphatase substrate (Sigma, St. Louis, MO) at room temperature. Plates were read at $405 \mathrm{~nm}$ using a PerkinElmer Victor 3 ELISA reader.

\section{C1q ELISA}

Murine monoclonal anti-human C1q (\#A201, Quide San Diego, CA) $(25 \mu \mathrm{l} / \mathrm{well}$ of $2 \mu \mathrm{g} / \mathrm{ml})$ in PBS was dry-coated into 96-well polystyrene microtiter plates (Costar, 3690, Corning) overnight at $4{ }^{\circ} \mathrm{C}$. Wells were blocked $3 \%$ nonfat dry milk with 50ul/well (\# M0841, LabScientific Highlands, NJ) in PBS for $4 \mathrm{~h}$ at room temperature. After rinsing the wells three times with PBS-0.05\%Tween, $25 \mu \mathrm{l}$ of serum samples diluted in PBS were added to each well. The serum dilutions were obtained by first making a 1:100 dilution and serially re-diluting this solution until it was 1 : 10,000 . Samples were incubated overnight at $4{ }^{\circ} \mathrm{C}$. Wells were then washed 3 times with PBS- Tween. Goat antiserum to human C1q (\#A301, Quidel) was diluted 1:1000 in $0.3 \%$ non-fat milk in PBS and added $(25 \mu \mathrm{l} /$ well $)$ for $2 \mathrm{~h}$ at room temperature. After washing 3 times in PBSTween, plates were incubated for $1 \mathrm{~h}$ at room temperature with rabbit anti-goat IgG antibody conjugated to alkaline phosphatase (\#A-4062, Sigma) diluted in 0.3\% non-fat milk in PBS at 1:500. The wells were washed 3 times with PBS-
Tween and incubated with $50 \mu \mathrm{l}$ of alkaline phosphatase substrate (Sigma) in solution (.5 M Na2CO3 and .01 M $\mathrm{MgCl} 2$ ) (check). The absorbance of each well was read at $30 \mathrm{~min}$ at $405 \mathrm{~nm}$. The standard curve of purified human $\mathrm{C} 1 \mathrm{q}$ was linear in the $2 \mathrm{ng}$ to $250 \mathrm{ng}$ range. Both the standards and serum samples were assayed in triplicate.

\section{Statistical methods}

The primary objective was to compare potential biomarkers of SLE among women grouped by risk for SLE based on race and malaria exposure: healthy Caucasian $(\mathrm{CHC})$ and African American (AAHC) women, African women with past exposure to malaria (MAL), unaffected sisters of lupus patients (SIS), and lupus patients (SLE). Since a high IgG:IgM anti-dsDNA antibody ratio and a low level of C1q are associated with SLE, and a low IgG: IgM anti-dsDNA antibody ratio and high level of C1q are associated with healthy controls, the LRI was calculated by $\frac{\operatorname{Ig} G}{\operatorname{Ig} \times \mathrm{Cl}^{2} \mathrm{~g}}$. For this analysis, original measurement units were used and plotted on log axis which resulted in data that were consistent with the usual assumptions of normality and equal variance across groups. One-way analysis of variance was used to compare each of these five markers separately across the groups. Upon finding a significant difference, Tukey's method of pairwise comparisons was used, separately for each marker, to determine which groups' means differed from one another on that marker. All statistical tests, including the Tukey test, were performed at the $5 \%$ significance level.

\section{Results}

\section{Anti-dsDNA antibodies}

As IgM antibodies precede the generation of IgG antibodies and protect against SLE onset, we assessed IgM anti-DNA antibodies in all 5 cohorts (Fig. 1). Titers were lowest in the SLE, SIS, and AAHC cohorts. Titers were significantly higher in the $\mathrm{CHC}$ cohort and highest in the MAL cohort.

We next assessed IgG anti-DNA antibodies in all cohorts (Fig. 2). CHC, AAHC and SIS had similar titers of these antibodies. The MAL cohort exhibited significantly increased IgG anti- dsDNA titers and the SLE cohort exhibited the highest titers.

\section{lgG:IgM ratio}

While significant differences in IgG and IgM anti-DNA titers were present, we reasoned that IgM and IgG antibodies compete for antigen, leading us to ask whether the IgG: IgM ratio was more critical to disease progression than the titer of either alone (Fig. 3). As expected, the SLE cohort had the highest ratio compared to all other cohorts. The MAL, SIS and AAHC cohorts had an intermediate ratio while the $\mathrm{CHC}$ cohort had the lowest ratio. 


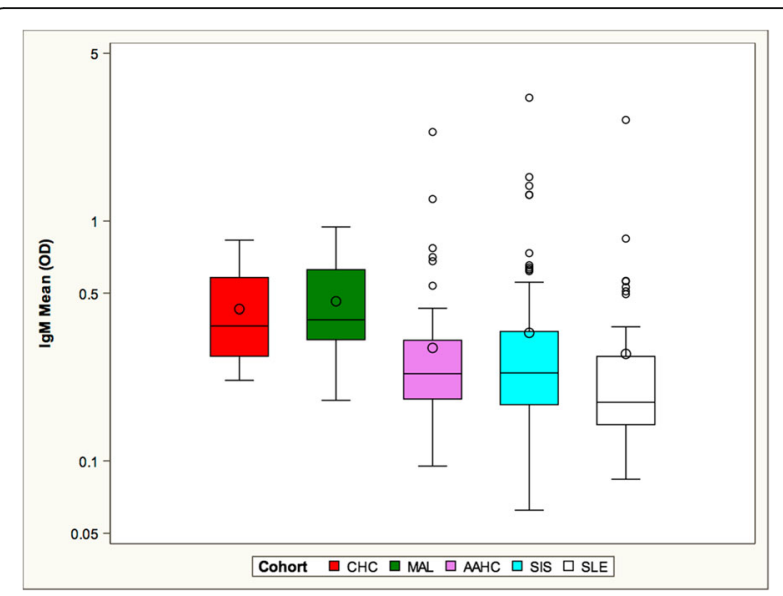

Fig. 1 The cohorts are organized in order of the presumed risk from lowest (left) to highest (right). The MAL cohort had the highest mean IgM anti-DNA level, followed by the CHC cohort. The mean of the SIS cohort did not differ from the mean of the AAHC and the SLE cohorts

\section{C1q levels}

C1q levels were assessed in all cohorts (Fig. 4). Not only is $\mathrm{C} 1 \mathrm{q}$ deficiency among the greatest risk factors for SLE, but C1q inversely correlates with disease activity (Horak et al. 2006). Anti-C1q antibodies have also correlated with disease activity (Bock et al. 2015). C1q levels were lowest in the SLE cohort, slightly higher but still low in the SIS cohort, intermediate within the $\mathrm{CHC}$ and AAHC cohorts and highest in individuals exposed to malaria, the MAL cohort.

\section{Lupus risk index}

Based on the putative protection conferred by a low IgG/IgM anti-dsDNA antibody ratio and a high C1q level, the LRI was developed to measure propensity for development of SLE for each individual (Fig. 5). The LRI

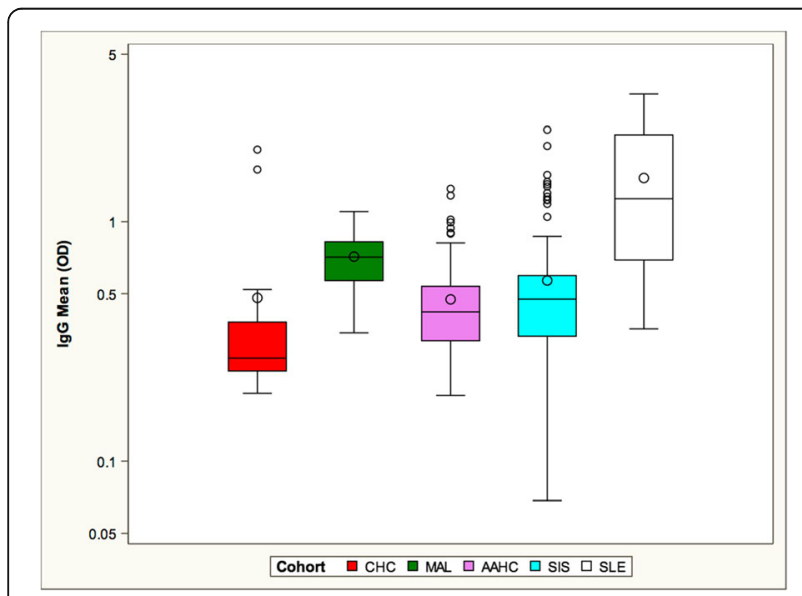

Fig. 2 The SLE and MAL cohort had significantly higher mean IgG anti-DNA levels than all other cohorts. The $\mathrm{CHC}$, AAHC and SIS cohorts did not differ from one another and had lower titers than the MAL and SLE cohorts

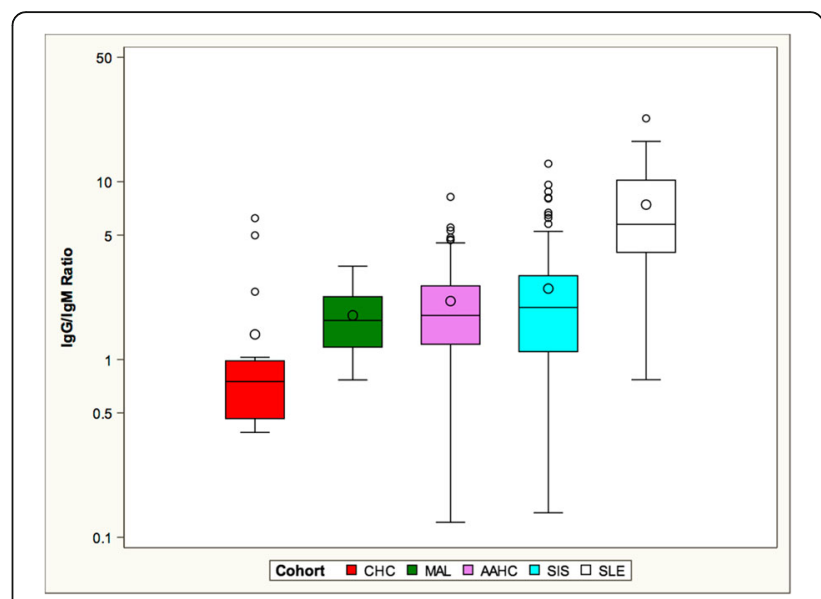

Fig. 3 SLE had the highest mean lgG/lgM anti-DNA antibody ratio. The mean ratios of the SIS, AAHC, and MAL cohorts did not significantly differ from each other. The mean ratio for the $\mathrm{CHC}$ cohort was significantly lower than all other groups

was defined as $\frac{\operatorname{IgG}}{\operatorname{IgM} \times \mathrm{Clq} q}$. The SLE patients exhibited the highest mean LRI, followed by the SIS cohort, and then the AAHC cohort, while the $\mathrm{CHC}$ and the MAL cohorts exhibited the lowest LRI.

\section{Discussion}

In this study, we examined serologic markers in 5 cohorts with variable risk for SLE to understand pathways that might predispose to or prevent disease onset. As anticipated, we observed high titers of IgM anti-DNA antibodies in the MAL cohort and high titers of IgG antiDNA antibodies in the SLE cohort. Analysis of the IgG/ IgM anti-DNA antibody ratio showed a high ratio in SLE patients, a low ratio in the $\mathrm{CHC}$ cohort and intermediate ratios within the SIS, MAL and AAHC cohorts.

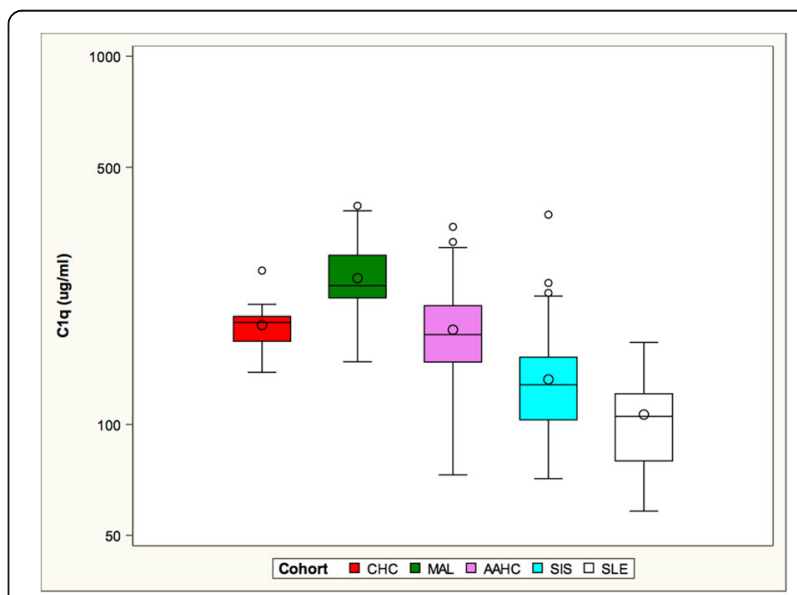

Fig. 4 The MAL cohort had the highest mean C1q level. The mean Clq levels of the $\mathrm{CHC}$ and $\mathrm{AAHC}$ cohorts did not differ from each other. The SIS cohort had a lower mean C1q level and the SLE cohort had the lowest level 


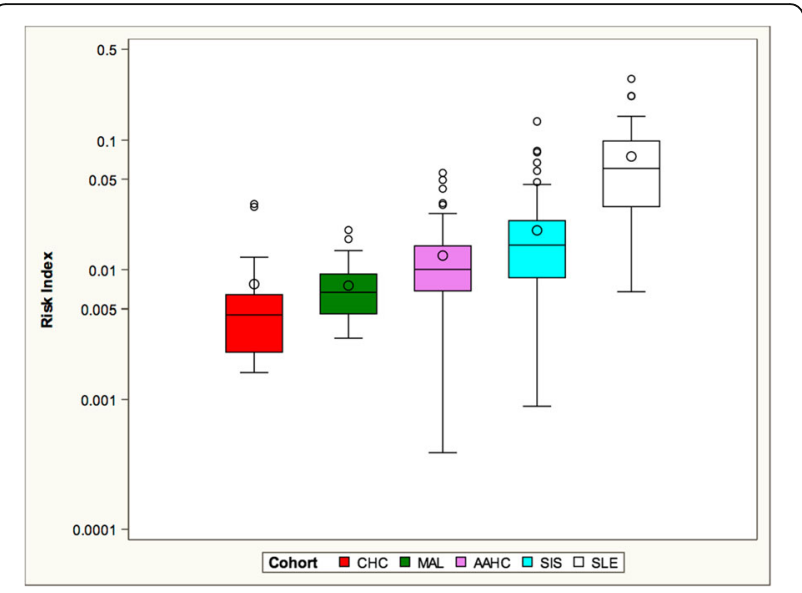

Fig. 5 All cohorts were significantly different from each other, except the $\mathrm{CHC}$ and MAL cohorts which showed no significant difference

The protective properties of IgM antibodies are known. IgM immune complexes engage $\mathrm{Clq}$ which will bind LAIR-1, an inhibitory surface receptor on hematopoietic cells (Son and Diamond 2015). IgM precedes IgG anti-dsDNA antibodies in mouse models of SLE and it has been shown in the $N Z B / W$ model that administration of IgM anti-DNA antibody will delay onset of disease. IgM, especially pentameric IgM, competes with IgG for antigen and thereby diminishes the load of IgG immune complexes including IgG anti-DNA immune complexes that bind to activating Fc receptors on myeloid cells to initiate an inflammatory cascade. Consistent with the model that IgM is protective against autoimmunity and IgG engages inflammatory pathways, mice genetically engineered to secrete IgG but not IgM will develop SLE (Marshak-Rothstein 2006; Boes et al. 2000). Moreover, B6.Sle1 mice which carry the sle 1 risk locus from $N Z M$ mice produce more antigen-specific $\operatorname{IgG}$ and total $\operatorname{IgG}$ and exhibit enhanced $\operatorname{IgM}$ to $\operatorname{IgG}$ class switching (Rahman et al. 2007), suggesting that part of the genetic risk for SLE may include a propensity to high IgG levels.

Malaria exposed individuals harbor anti-nuclear antibodies, some of which cross react with malarial antigens. The ANA pattern in malaria is different from patterns observed in SLE, suggesting fine specificity differences, but anti-DNA antibodies have been reported (Hommel et al. 2014; Hirako et al. 2015). That these anti-DNA antibodies are primarily IgM is consistent with reports of high IgM antibodies in response to malarial infection (Pleass et al. 2016; Czajkowsky et al. 2010). Interestingly, the Fulani population in Mali experiences less severe malarial disease than the Dogon population; IgM anti-malarial titers are higher in the Fulani than the Dogon and may in part account for the less severe disease (Maiga et al. 2013). Why malaria exposure leads to high IgM levels and whether this reflects activation of "innate" B1 or marginal zone B cells or impaired class switching in malaria patients is not known, but may relate to high BAFF levels which are seen in malaria exposed individuals (Scholzen and Sauerwein 2013).

We analyzed serum levels of $\mathrm{Clq}$ as low $\mathrm{C} 1 \mathrm{q}$ correlates with disease severity and absence of $\mathrm{C} 1 \mathrm{q}$ is a strong genetic risk factor for SLE. C1q opsonizes apoptotic cells to remove debris in a non-inflammatory fashion in an IgMmediated pathway. C1q binds the collagen receptor LAIR1 through its collagen-like tail to maintain monocyte quiescence and prevent monocyte to DC differentiation (Son et al. 2012). The interaction of C1q with LAIR- 1 prevents activation of endosomal TLRs in DCs by nucleic acid ligands. Finally, C1q blocks the transfer of an IFN signature transfer to healthy PBMCs by SLE serum. Thus, IgM antibodies function in conjunction with $\mathrm{C} 1 \mathrm{q}$ to mitigate inflammatory pathways.

As expected, C1q levels were diminished in the SLE, and, to a lesser degree, in the SIS cohort. There was no difference between the $\mathrm{CHC}$ and $\mathrm{AAHC}$ cohorts. $\mathrm{Clq}$ levels were highest in the MAL cohort. Mechanisms increasing serum $\mathrm{C} 1 \mathrm{q}$ levels are unknown but $\mathrm{Clq}$ is produced by anti-inflammatory M2-like macrophages (Fraser et al. 2015). While these have not been specifically shown to be increased in malaria infection, they are increased by helminthic infections (Fairweather and Cihakova 2009). Elevated $\mathrm{C} 1 \mathrm{q}$ may also relate to the binding of IgM to Pfem1, a molecule expressed on the membrane of parasite-infected erythrocytes. The interaction of IgM with Pfem prevents the binding of IgM to C1q and may thus raise levels of soluble C1q (Czajkowsky et al. 2010). Based on the IgG:IgM anti-DNA antibody ratio and the $\mathrm{Clq}$ level, we generated an LRI. This score confirmed the known risk of SLE; the highest LRI was present in the SLE cohort. Among the non-SLE cohorts, LRI was highest in SIS followed by AAHC, while the $\mathrm{CHC}$ and MAL cohorts exhibited the lowest LRI. Although the MAL cohort exhibited relatively high IgG anti-dsDNA antibody titers, the high IgM antidsDNA antibody and C1q levels reduced the LRI. These serologic features may contribute to the protection malaria confers against the development of SLE. Understanding how malaria, even when recurrent, blocks the IgM to IgG switch has important therapeutic implications.

\section{Conclusion}

In summary, we have studied populations with different risk for developing SLE to propose a metric to assess that risk. A risk score is as robust as its components are pathophysiologically relevant. DsDNA IgG, IgM and C1q, which are the components of the LRI that we propose, are known to respond to changes in disease activity. A tool such as this that can predict the risk of developing clinical SLE 
would be useful to assess the effectiveness of early interventions. Therapy with hydroxychloroquine, for example, delays disease onset (Virdis et al. 2015); we would anticipate that its therapeutic effect would be reflected in the LRI. Longitudinal studies, including in our unique sisters cohort are needed to validate our findings. These observations additionally suggest new therapeutic approaches for the treatment of SLE.

\author{
Abbreviations \\ AAHC: Healthy African American women; CHC: Healthy Caucasian women; \\ LRI: Lupus risk index; MAL: Women from Mali with a history of malaria \\ infection; SIS: Unaffected sisters of SLE patients; SLE: Systemic lupus \\ erythematosus
}

\section{Funding}

This study was supported by a grant from the Lupus Research Institute and the New York State Department of Health, Empire Clinical Research Investigator Program.

\section{Availability of data and materials}

The data that support the findings of this study are available from the corresponding author upon request.

\section{Authors' contributions}

Study conception and design: BD Sample acquisition: CA, MM, OD, AT, PG Analysis and interpretation of data: JB, BT, ML, KP Drafting of manuscript: JB, $B D, K P, M L, C A, M M$ Critical revision: BD. All authors read and approved the final manuscript.

\section{Ethics approval and consent to participate}

The study was approved by the Institutional Review Board at the Northwell Health, Manhasset, NY and the Comité d'Ethique de la FMPOS, Bamako, Mali.

\section{Competing interests}

The authors declare that they have no competing interests.

\section{Publisher's Note}

Springer Nature remains neutral with regard to jurisdictional claims in published maps and institutional affiliations.

\section{Author details \\ ${ }^{1}$ The Feinstein Institute for Medical Research, Center for Autoimmune, Musculoskeletal and Hematopoietic Diseases, 350 Community Dr, Manhasset, NY 11030, USA. ${ }^{2}$ Department of Statistical Science, Cornell University, Ithaca, NY, USA. ${ }^{3}$ Department of Internal Medicine, Istanbul University, Istanbul, Turkey. ${ }^{4}$ The Feinstein Institute for Medical Research, Center for Genomics and Human Genetics, Manhasset, NY, USA. ${ }^{5}$ Malaria Research and Training Center, Bamako, Mali. ${ }^{6}$ Deputy of the Department of Internal Medicine, University Hospital, Bamako, Mali. ${ }^{7}$ The Feinstein Institute for Medical Research, Center of Biostatistics Unit Manhasset, Manhasset, NY, USA. ${ }^{8}$ UCLA David Geffen School of Medicine, Los Angeles, CA 90095, USA. ${ }^{9}$ University of Chicago Medical Center, Chicago, IL, USA. ${ }^{10}$ Hospital for Sick Children, University of Toronto, Toronto, ON M5G 1X8, Canada. ${ }^{11}$ University of Michigan, Ann Arbor, MI 48109, USA. ${ }^{12}$ Rush University Medical Center, Chicago, IL 60612, USA. ${ }^{13}$ Cedars Sinai Medical Center, Los Angeles, CA 90048, USA. ${ }^{14}$ Instituto Nacional de Ciencias Medicas y Nutrician Salvador Zubiran, Mexico City, Mexico.}

Received: 2 April 2018 Accepted: 3 April 2018

Published online: 01 June 2018

\section{References}

Arbuckle MR, et al. Development of autoantibodies before the clinical onset of systemic lupus erythematosus. N Engl J Med. 2003;349:1526-33.

Bock M, Heijnen I, Trendelenburg M. Anti-C1q antibodies as a follow-up marker in SLE patients. PLoS One. 2015;10:e0123572.
Boes $M$, et al. Accelerated development of lgG autoantibodies and autoimmune disease in the absence of secreted IgM. Proc Natl Acad Sci U S A. 2000;97:1184-9.

Clatworthy MR, et al. Systemic lupus erythematosus-associated defects in the inhibitory receptor FcgammaRllb reduce susceptibility to malaria. Proc Natl Acad Sci U S A. 2007;104:7169-74.

Czajkowsky DM, et al. IgM, Fcu-receptors, and malarial immune evasion. J Immunol. 2010;184:4597-603.

Daniel-Ribeiro CT, Zanini G. Autoimmunity and malaria: what are they doing together? Acta Trop. 2000;76:205-21.

Deane KD, El-Gabalawy H. Pathogenesis and prevention of rheumatic disease: focus on preclinical RA and SLE. Nat Rev Rheumatol. 2014;10:212-28.

Ehrenstein MR, Cook HT, Neuberger MS. Deficiency in serum immunoglobulin (lg)M predisposes to development of IgG autoantibodies. J Exp Med. 2000;191:1253-8.

Fairweather D, Cihakova D. Alternatively activated macrophages in infection and autoimmunity. J Autoimmun. 2009;33:222-30.

Floto RA, et al. Loss of function of a lupus-associated FcgammaRllb polymorphism through exclusion from lipid rafts. Nat Med. 2005;11:1056-8.

Fraser D, Melzer E, Camacho A, Gomez M. Macrophage production of innate immune protein $\mathrm{C} 1 \mathrm{q}$ is associated with M2 polarization (INM1P.434). J Immunol. 2015;194:56.11

George A, Ogunbiyi A. Systemic lupus erythematosus: a rarity in West Africa, or a yet to be investigated entity. Lupus. 2005;14:924-5.

Gilkeson G, et al. The United States to Africa lupus prevalence gradient revisited. Lupus. 2011;20:1095-103.

Greenwood BM. Autoimmune disease and parasitic infections in Nigerians. Lancet. 1968;2:380-2.

Greenwood BM, Herrick EM, Voller A. Suppression of autoimmune disease in NZB and (NZB x NZW) F1 hybrid mice by infection with malaria. Nature. 1970;226:266-7.

Gronwall C, Silverman GJ. Natural IgM: beneficial autoantibodies for the controlof inflammatory and autoimmune disease. J Clin Immunol. 2014;34(Suppl 1):S12-21.

Gronwall C, Vas J, Silverman GJ. Protective roles of natural IgM antibodies. Front Immunol. 2012;3:66.

Hirako IC, et al. DNA-containing Immunocomplexes promote Inflammasome assembly and release of pyrogenic cytokines by CD14+ CD16+ CD64high CD32low inflammatory monocytes from malaria patients. MBio. 2015;6:e01605-15.

Hoffman W, Lakkis FG, Chalasani G. B cells, antibodies, and more. Clin J Am Soc Nephrol. 2016;11:137-54.

Hommel B, et al. Chronic malaria revealed by a new fluorescence pattern on the antinuclear autoantibodies test. PLoS One. 2014;9:e88548.

Horak P, et al. C1 q complement component and -antibodies reflect SLE activity and kidney involvement. Clin Rheumatol. 2006;25:532-6.

Jiang $Y$, et al. Genetically determined aberrant down-regulation of FcgammaRIIB1 in germinal center B cells associated with hyper-lgG and lgG autoantibodies in murine systemic lupus erythematosus. Int Immunol. 1999;11:1685-91.

Linnik MD, et al. Relationship between anti-double-stranded DNA antibodies and exacerbation of renal disease in patients with systemic lupus erythematosus. Arthritis Rheum. 2005;52:1129-37.

Maiga B, et al. Human candidate polymorphisms in sympatric ethnic groups differing in malaria susceptibility in Mali. PLoS One. 2013:8:e75675.

Manderson AP, Botto M, Walport MJ. The role of complement in the development of systemic lupus erythematosus. Annu Rev Immunol. 2004;22:431-56.

Marshak-Rothstein A. Toll-like receptors in systemic autoimmune disease. Nat Rev Immunol. 2006;6:823-35.

McGill PE, Oyoo GO. Rheumatic disorders in sub-saharan Africa. East Afr Med J. 2002;79:214-6.

Molokhia M, McKeigue P. Risk for rheumatic disease in relation to ethnicity and admixture. Arthritis Res. 2000;2:115-25.

Molokhia M, McKeigue PM, Cuadrado M, Hughes G. Systemic lupus erythematosus in migrants from West Africa compared with afro-Caribbean people in the UK. Lancet. 2001;357:1414-5.

Molokhia M, et al. Relation of risk of systemic lupus erythematosus to west African admixture in a Caribbean population. Hum Genet. 2003;112:310-8.

Pavlovic M, et al. Pathogenic and epiphenomenal anti-DNA antibodies in SLE. Autoimmune Dis. 2010;2011:462841.

Pleass RJ, Moore SC, Stevenson L, Hviid L. Immunoglobulin M: restrainer of inflammation and mediator of immune evasion by Plasmodium falciparum malaria. Trends Parasitol. 2016;32:108-19.

Pritchard NR, et al. Autoimmune-prone mice share a promoter haplotype associated with reduced expression and function of the fc receptor FcgammaRII. Curr Biol. 2000;10:227-30. 
Rahman ZS, et al. Expression of the autoimmune Fcgr2b NZW allele fails to be upregulated in germinal center B cells and is associated with increased $\lg G$ production. Genes Immun. 2007:8:604-12.

Ramos PS, Brown EE, Kimberly RP, Langefeld CD. Genetic factors predisposing to systemic lupus erythematosus and lupus nephritis. Semin Nephrol. 2010;30:164-76.

Scholzen A, Sauerwein RW. How malaria modulates memory: activation and dysregulation of B cells in Plasmodium infection. Trends Parasitol. 2013;29: 252-62.

Son M, Diamond B. C1q-mediated repression of human monocytes is regulated by leukocyte-associated Ig-like receptor 1 (LAIR-1). Mol Med. 2015;20:559-68.

Son M, Diamond B, Santiago-Schwarz F. Fundamental role of C1q in autoimmunity and inflammation. Immunol Res. 2015;63:101-6.

Son M, Santiago-Schwarz F, Al-Abed Y, Diamond B. C1q limits dendritic cell differentiation and activation by engaging LAIR-1. Proc Natl Acad Sci U S A. 2012:109:E3160-7.

Teruel M, Alarcon-Riquelme ME. The genetic basis of systemic lupus erythematosus: what are the risk factors and what have we learned. J Autoimmun. 2016;74:161-75

Virdis A, et al. Early treatment with hydroxychloroquine prevents the development of endothelial dysfunction in a murine model of systemic lupus erythematosus. Arthritis Res Ther. 2015;17:277.

Werwitzke $\mathrm{S}$, et al. Inhibition of lupus disease by anti-double-stranded DNA antibodies of the lgM isotype in the (NZB x NZW)F1 mouse. Arthritis Rheum. 2005:52:3629-38.

Wozencraft AO, Lloyd CM, Staines NA, Griffiths VJ. Role of DNA-binding antibodies in kidney pathology associated with murine malaria infections. Infect Immun. 1990;58:2156-64.

Zomalheto Z, et al. Pattern of systemic lupus erythematosus in Benin and west African patients. La Tunisie Medicale. 2014;92:707-10.

\section{Ready to submit your research? Choose BMC and benefit from:}

- fast, convenient online submission

- thorough peer review by experienced researchers in your field

- rapid publication on acceptance

- support for research data, including large and complex data types

- gold Open Access which fosters wider collaboration and increased citations

- maximum visibility for your research: over $100 \mathrm{M}$ website views per year

At BMC, research is always in progress.

Learn more biomedcentral.com/submissions 